\title{
Role of Technology for the Management of AKI in Critically III Patients: From Adoptive Technology to Precision Continuous Renal Replacement Therapy
}

\author{
J. Cerdáa ${ }^{\text {I. Baldwin }}{ }^{c}$ P.M. Honore ${ }^{d} \quad$ G. Villa ${ }^{\text {e John A. Kellum }}{ }^{b}$ \\ Claudio Ronco $^{f}$ on behalf of the ADQI Consensus Group
}

\begin{abstract}
${ }^{a}$ Department of Medicine, Albany Medical College, Albany N.Y., and ${ }^{\mathrm{b}}$ Department of Critical Care Medicine, Center for Critical Care Nephrology, University of Pittsburgh School of Medicine, Pittsburgh, Pa., USA; 'Department of Intensive Care, Austin Hospital, Melbourne, Australia; ${ }^{d}$ Department of Intensive Care, Universitair Ziekenhuis Brussel, VUB

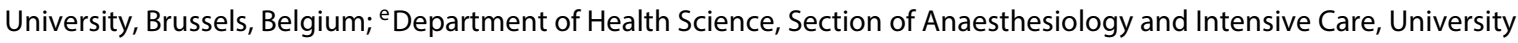
of Florence, Florence, and fDepartment of Nephrology and International Renal Research Institute of Vicenza (IRRIV), San Bortolo Hospital, Vicenza, Italy
\end{abstract}

\section{Key Words}

Continuous renal replacement therapy - Technology · Dialysis machine - Electronic medical record · Acute kidney injury - Extracorporeal therapies · Continuous veno-venous hemofiltration . Continuous veno-venous hemodialysis - Continuous veno-venous hemodiafiltration . Hemoperfusion - Plasma exchange - Treatment adequacy . Dose of dialysis · Sepsis - Multiple organ failure - ECCOR . ECMO $\cdot$ Precision CRRT

Bagshaw S.M., Critical Care Medicine, Faculty of Medicine and Dentistry, University of Alberta, Edmonton, Canada; Chakravarthi R.M., Department of Nephrology, STAR Hospitals, Hyderabad, India; De Rosa S., International Renal Research Institute of Vicenza (IRRIV), San Bortolo Hospital, Vicenza, Italy; Floris M., Renal Unit, Ospedale 'G. Brotzu', Cagliari, Italy; Hoste E.A.J., Ghent University Hospital, Ghent University, Ghent, Belgium; Research Foundation Flanders, Brussels, Belgium; Joannidis M., Division of Intensive Care and Emergency Medicine, Department of Internal Medicine, Medical University Innsbruck, Innsbruck, Austria; Mehta R.L., University of California San Diego, San Diego, California; Murugan R., Department of Critical Care Medicine, Center for Critical Care Nephrology, University of Pittsburgh School of Medicine, Pittsburgh, Pa., USA; Neri M., International Renal Research Institute of Vicenza (IRRIV), San Bortolo Hospital, Vicenza, Italy; Pani A., Renal Unit,

\begin{abstract}
This paper reports on the continuous renal replacement therapy (CRRT) technology group recommendations and research proposals developed during the 17th Acute Dialysis Quality Initiative Meeting in Asiago, Italy. The group was tasked to address questions related to the impact of technology on acute kidney injury management. We discuss technological aspects of the decision to initiate CRRT and the components of the treatment prescription and delivery, the
\end{abstract}

Ospedale 'G. Brotzu', Cagliari, Italy; Osterman M., Guy's and St. Thomas' Hospital, London, UK; Ricci Z., Department of Pediatric Cardiology and Cardiac Surgery, Ospedale Pediatrico Bambino Gesù IRCCS, Roma, Italy; Rosner M., Department of Medicine, University of Virginia, Charlottesville, Va., USA; Samoni S., Institute of Life Sciences, Sant'Anna School of Advanced Studies, Pisa, Italy; Tolwani A.J., Department of Nephrology, University of Alabama at Birmingham, Ala., USA; Xiaoqiang Ding, Department of Nephrology, Shanghai Institute of Kidney and Dialysis, Zhongshan Hospital, Fudan University, Shanghai, China; Wuhua Jiang, Department of Nephrology, Shanghai Institute of Kidney and Dialysis, Zhongshan Hospital, Fudan University, Shanghai, China.

Report from the 17th Acute Disease Quality Initiative (ADQI) Consensus Conference. Asiago, Italy, June 10-13, 2016.

\section{KARGER}

E-Mail karger@karger.com

www.karger.com/bpu (c) 2016 S. Karger AG, Basel

0253-5068/16/0423-0248\$39.50/0
Professor Claudio Ronco

Department of Nephrology and International Renal Research

Institute of Vicenza, San Bortolo Hospital

IT-36100 Vicenza (Italy)

E-Mail cronco@goldnet.it 
integration of information technology (IT) on overall patient management, the incorporation of CRRT into other 'non-renal' extracorporeal technologies such as ECMO and ECCO ${ }^{2} \mathrm{R}$ and the use of sorbents in sepsis and propose new areas for future research. Instead of reviewing current knowledge, the group focused on developing a renovated research agenda that reflects current and future technological advances, centered on innovations in new equipment, membranes and IT that will permit the integration of patient care and decisionmaking processes for years to come.

(c) 2016 S. Karger AG, Basel

\section{Introduction}

In 1977, Peter Kramer first described a new treatment modality for acute kidney injury (AKI) defined as continuous arteriovenous (AV) hemofiltration. The technique consisted of the extended use of a pumpless extracorporeal circuit connected to an artery and a vein, whereby blood flow was maintained by the AV pressure gradient [1]. Subsequently, the use of double lumen catheters allowed the development of continuous veno-venous hemofiltration $(\mathrm{CVVH})$ where a blood pump often borrowed from chronic dialysis equipment and a rudimentary fluid balance system delivered higher blood flows and fluid exchange volumes [2]. Almost 15 years later, different continuous renal replacement (extracorporeal) therapies (CRRT) such as CVVH, continuous veno-venous hemodialysis (CVVHD) and continuous veno-venous hemodiafiltration (CVVHDF), respectively were made available by the launch of dedicated integrated equipment with improved capabilities and improved safety [3]. In the following years, several technological advances were made leading to the development of second-, third- and fourth-generation CRRT machines [4]. Since then, CRRT remains a continuously evolving modality, with new applications of current devices and innovative future possibilities.

In 2000, the Acute Dialysis Quality Initiative (ADQI) was created by a group of experts that gathered in New York for the first consensus conference. The theme was CRRT, and results were published as a conference report and practice recommendation [5]. Several conferences have since been held in different parts of the world leading to important achievements on definitions, classification and treatment of AKI and the cardiorenal syndrome. ADQI and its website have become a reference for education and a repository of knowledge [6-8] and the incubator for new ideas, as well as the site where new studies and trials are suggested or designed.

Role of Technology in CRRT for the Management of Critically Ill Patients
After tackling numerous themes in the field of AKI over the years, the founders felt a compelling need to revisit and update what was presented and published in the first 2000 consensus report; so a new conference on CRRT was organized in Asiago, Italy, for this purpose. Four themes were established in the conference: CRRT indications and patient selection, solute control and treatment dose, CRRT technology and fluid management. This paper reports on the research questions, resolutions and statements agreed upon by the expert group in the area of CRRT technology, as well as the research agenda for years to come.

\section{Methods}

The ADQI process has been described previously and complete ADQI methodology description is available at www.adqi. org. Briefly, we convened a 3-day consensus conference in Asiago, Italy, in June 2016. We invited experts in nephrology and critical care medicine from both adult medicine and pediatrics. Principles of current practice were debated in light of existing evidence. Areas of broad consensus were described and a research agenda was developed using a modified Delphi method. Prior to the conference, we identified fundamental questions for which consensus would be sought. During the pre-conference phase, each workgroup performed comprehensive literature searches to summarize existing knowledge and to identify gaps that could be addressed by future research. Core questions/concepts were crafted and these were presented to the entire ADQI consensus group during the conference; a series of breakout sessions and plenary presentations allowed debate, discussion and refinement of these concepts. For the timing and patient selection workgroup, literature searches were conducted using the following terms: 'renal replacement therapy' together with either 'acute kidney injury', 'acute renal failure' or 'continuous veno-venous hemodialysis/hemofiltration', in MEDLINE using PubMed as the search engine. The 4 core questions ultimately addressed by the workgroup are shown in table 1 .

\section{Research Questions and Consensus Statements}

The group identified several CRRT technology topics requiring a collegial discussion and consensus. Given the wide availability of published evidence in the field, the group focused on developing a renovated research agenda reflecting current and future technological needs and expected advances, centering on new equipment, membranes and information technology (IT) integrating patient care and the decision-making process.

Question 1: How and when does technology support AKI management? 
Table 1. Questions identified by the consensus group

(1) How and when does technology support AKI management?

(2) Should initiation of CRRT be supported by a consistent decision algorithm?

(3) What are the essential technological components of the CRRT prescription?

(4) What are the essential technological elements necessary for CRRT delivery?

(5) Can technology assist in the process of prescription and dynamic re-evaluation of physiological therapy targets?

(6) How should adequacy and dose delivery be prescribed?

(7) When should 'other' techniques be prescribed?

(8) What are the main areas of future research in CRRT technology?

- Throughout AKI management, technology is involved at different levels and contributes to improve practice and patient outcomes by supporting prescription and delivery of CRRT.

- AKI management is a continuum from detection to treatment, which must include continuous re-evaluation of treatment prescription and delivery. The use of modern IT tools is recommended to improve practice and patient care (Grade A).

- Integration of information communication technology tools in the practice of CRRT represents the most important technological advances to be implemented and studied.

A multidisciplinary effort is essential to identify patients at risk of developing AKI [9]. In spite of new epidemiological information, marginal or no advances have been made to reduce the incidence and severity of AKI. Thus, at every point in the AKI continuum, clinicians must take advantage of new technology to improve clinical care and patient outcome (fig. 1).

New biomarkers for risk identification and early AKI diagnosis are today available, both as laboratory and point of care measurements. Moreover, nowadays practitioners may use multiple alternative technological resources for the management of critically ill patients, such as monitors for hemodynamic optimization, bioimpedance vectorial analysis and non-invasive imaging techniques. Identification of patients susceptible to develop AKI and detection of subclinical AKI before progression to kidney dysfunction may reduce progression toward clinical AKI, diminish the severity of the kidney insult and improve patient outcome [10]. Early identification of clinical AKI may allow the application of protective measures and suitable management, geared to reduce pro- gression and improve renal recovery [11]. Several electronic alert systems mainly centered, but not limited, to changes in serum creatinine and/or urine output have been proposed to prompt the use of early management strategies [12]. These systems, based on algorithms derived from continuously fed electronic medical records (EMRs), generate a decision support pathway that may help clinicians to identify when CRRT is indicated. Such early intervention defines the targets of renal replacement therapy (RRT) in terms of solute removal, fluid balance, homeostatic corrections and/or requirement for multiple organ support.

The appropriate use of modern CRRT equipment ensures a safe and effective treatment. The features of lastgeneration CRRT machines allow easy management of different treatment modalities (diffusive $=$ CVVHD; convective $=\mathrm{CVVH}$; mixed CVVHDF, according to availability, local preferences and experience) to meet patient requirements [9]. New technological advances in anticoagulation, membranes and dialyzers and machine operational characteristics provide the basis for safe and correct management of specific clinical conditions and patient needs $[13,14]$.

In parallel with prescription, the delivery phase of CRRT is today supported by technological improvements in hardware and software that provide guidance in several aspects of the treatment including downtime dose compensation, calcium replacement during citrate anticoagulation, circuit pressure profiles and appropriate fluid balance [15-19]. Large screens on machines permit online tutorials and assisted troubleshooting.

Finally, the continuum of AKI management must include a feedback loop for prescription reassessment after monitoring, data collection and evaluation of the deliv- 


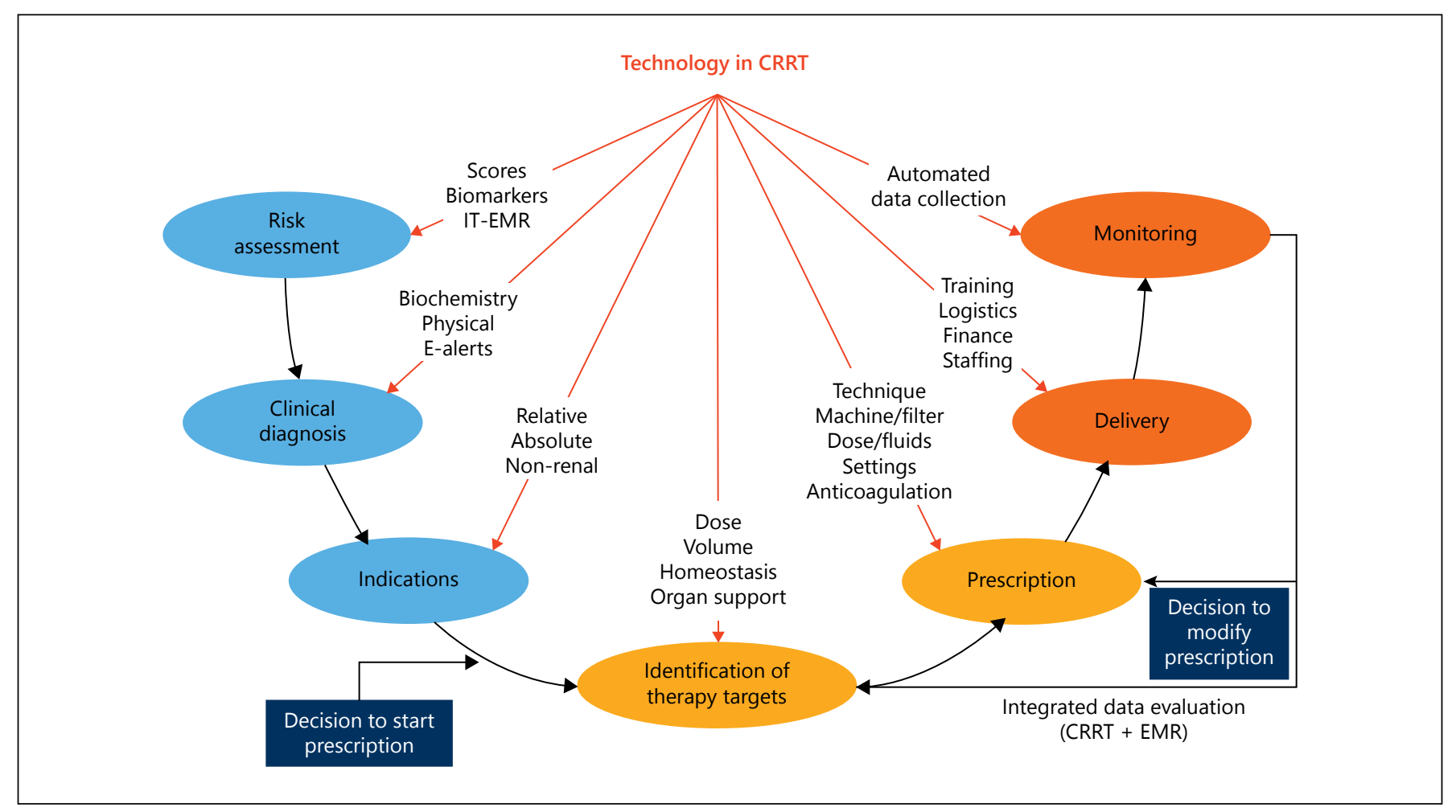

Fig. 1. The role of technology at different levels in the continuum of AKI management. Reprinted with permission from www.ADQI.org.

ered treatment. Monitoring and data collection systems based on various connectivity platforms (machine, local or cloud-based) help physicians to measure whether end points are achieved and support modifications of the initial CRRT prescription based on updated clinical targets. Timing and rapidity of feedback will depend on the frequency of data acquisition and evaluation. Patient cards or machine-derived records may be used to identify not only problems in single treatments, but also suboptimal policies and procedures in the entire center population. Moreover, online information from the CRRT machine will permit immediate feedback and proper setting modification. A biofeedback loop can either be automatic (as in modern chronic dialysis machines, where ultrafiltration and dialysate sodium concentration is automatically modified based on blood volume variation) or simply suggested on the machine screen. In this latter case, each feedback action must either be either authorized or manually implemented.

Question 2: Should initiation of CRRT be supported by a consistent decision algorithm?

- The process of patient evaluation and identification of therapy targets should lead to a 'precision' (personal- ized) prescription subject to change over time (not graded).

- This process should identify clear steps and shared terminology (published nomenclature) to enable easy pragmatic trials from populations, as well as delivery of precision CRRT for single patients (Grade A).

Timing and protocol for CRRT initiation has been addressed by the KDIGO acute dialysis guideline [20]. Several algorithms to structure this decision process have been proposed $[21,22]$. All these decision pathways implement immediate start of RRT in cases of lifethreatening conditions. It is generally accepted that hemodynamic stabilization is required, as well as frequent monitoring of acid-base status, electrolytes, fluids and other parameters. However, in the majority of cases, the decision to start RRT still predominantly relies on clinical judgment, utilizing unclear criteria and poorly defined timing.

A recent conceptual approach suggests the determination of an index that measures the ratio between the weight of the demands imposed on the kidneys and the estimated capacity of the impaired kidneys to respond to that demand. Whenever the demand exceeds the capaci- 
ty, CRRT is necessary [23]. As critically ill patients continuously vary in their status, assessment of the gap between demand and capacity may have to be continuously reassessed to assist in the decision to start the treatment.

In figure 2, we propose a simple effective algorithm to manage critically ill patients from admission to AKI resolution. The algorithm is based on a cascade of observations and actions with potential loops throughout the implementation of CRRT. This approach allows for a 'precise' CRRT prescription and management, and will also permit data collection at different gates of the algorithm to facilitate pragmatic trials. Data collection in EMR and CRF should be consistent with published harmonized nomenclature [15-19].

Questions 3, 4: What are the essential technological elements of the CRRT prescription? What are the essential technological components of CRRT delivery?

- CRRT prescription and delivery must include all the necessary components to proceed to prompt treatment implementation and delivery (not graded).

- Prescription should be made according to patient needs (demand/capacity gap) and desired physiological targets to allow precise CRRT initiation. Delivery should be as close to prescription as possible (Grade A).

- Prescription should be dynamic and frequently adjusted based on patient needs (Grade A). Ideally dynamic prescription requires a full treatment reassessment at least every $6 \mathrm{~h}$.

- Dedicated equipment (CRRT machines) should be used to deliver specific modalities; adoptive technologies should be avoided (Grade A).

- When prescribing CRRT, availability, training, environmental and staffing issues should be considered and prescription should be made according to local conditions and usage (Grade A).

Adequate RRT prescription requires a match between patient clinical characteristics and requirements, equipment availability and characteristics of the environment where the treatment is implemented (fig. 3) [2, 9].

Initial treatment targets require accurate assessment of patient severity of disease, comorbidities, multiple organ failure, fluid status, hemodynamics, homeostatic abnormalities, catabolism and impaired kidney functional capacity. Once treatment targets have been identified, decisions must be made regarding the best way to achieve the desired end points. Prescription must necessarily take into consideration the treatment feasibility, according to logistics and resources. Effective treatment delivery requires decisions on modality, operational characteristics (including membrane and filter characteristics), choice of solutions for replacement fluid and dialysate, as well as the decision on a prescribed dose. Table 2 lists all the necessary components to proceed to appropriate CRRT prescription and delivery.

Necessarily, all those decisions will occur in a singular hospital environment with specific characteristics, which will have an impact just as important as patient features and available equipment. Thus, availability of nursing personnel and their training, institutional support, quality of policies and procedures and decisions on cost will be key determinants of what can and cannot be done in each situation.

The use of latter generation CRRT machines will easily enable the performance of best quality treatments. In order to achieve best results, the use of RRT machines must be integrated into the care of the patient as a whole; in some patients, initiation of CRRT will be necessary to address the specific consequences of kidney failure (e.g., uremia or dyselectrolytemia) but in most cases, the use of RRT will assist in the management of multiple organ failures, where the start of RRT will help manage 'extra-renal' problems such as fluid overload resulting from heart failure or severe acidosis caused by intestinal ischemia. Those conditions are intrinsically unstable and rapidly variable and therefore, the prescription will need to change and adjust many times a day. Accordingly, it is preferable to plan and to adjust prescription in a frequent (optimally every $6 \mathrm{~h}$ ) than on a 24 -hour basis.

Timing of initiation of RRT and prescription should be made according to patient needs. In this manner, progress in the definition of the demands imposed onto the failing kidney and a measure of the capacity of the kidney to respond to the challenge should guide treatment [2325].

Given the interaction between different teams, easy and frequent communication is essential to achieve best results. Such interaction will require the use of a clearly defined common language, and therefore, the use of most recently harmonized terminology is recommended [15-19]. Moreover, progress in the area of technology will necessarily require interactions utilizing a common language to avoid confusion and to ensure appropriate measurement of results. Finally, the appropriate design of research questions and results makes the use of a common language unavoidable. Pragmatic trials should be implemented as soon as new technologies are applied, to close the loop and learn from successes and mistakes.

Ideal prescription should be made according to patient needs and desired physiological targets. In reality, local 


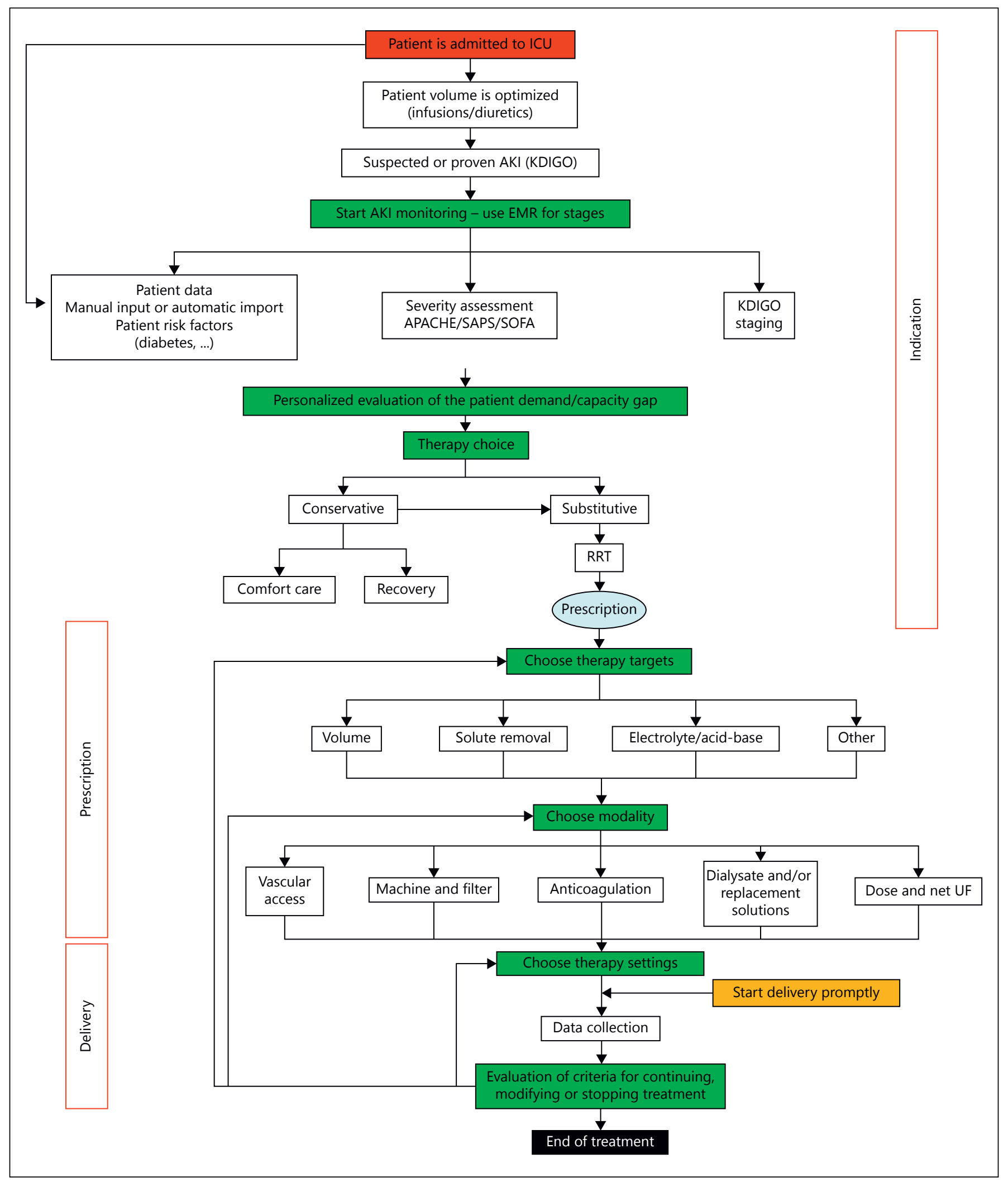

Fig. 2. Proposed algorithm to manage critically ill patients from AKI diagnosis to CRRT prescription, delivery and weaning. Reprinted with permission from www.ADQI.org.

Role of Technology in CRRT for the Management of Critically Ill Patients 


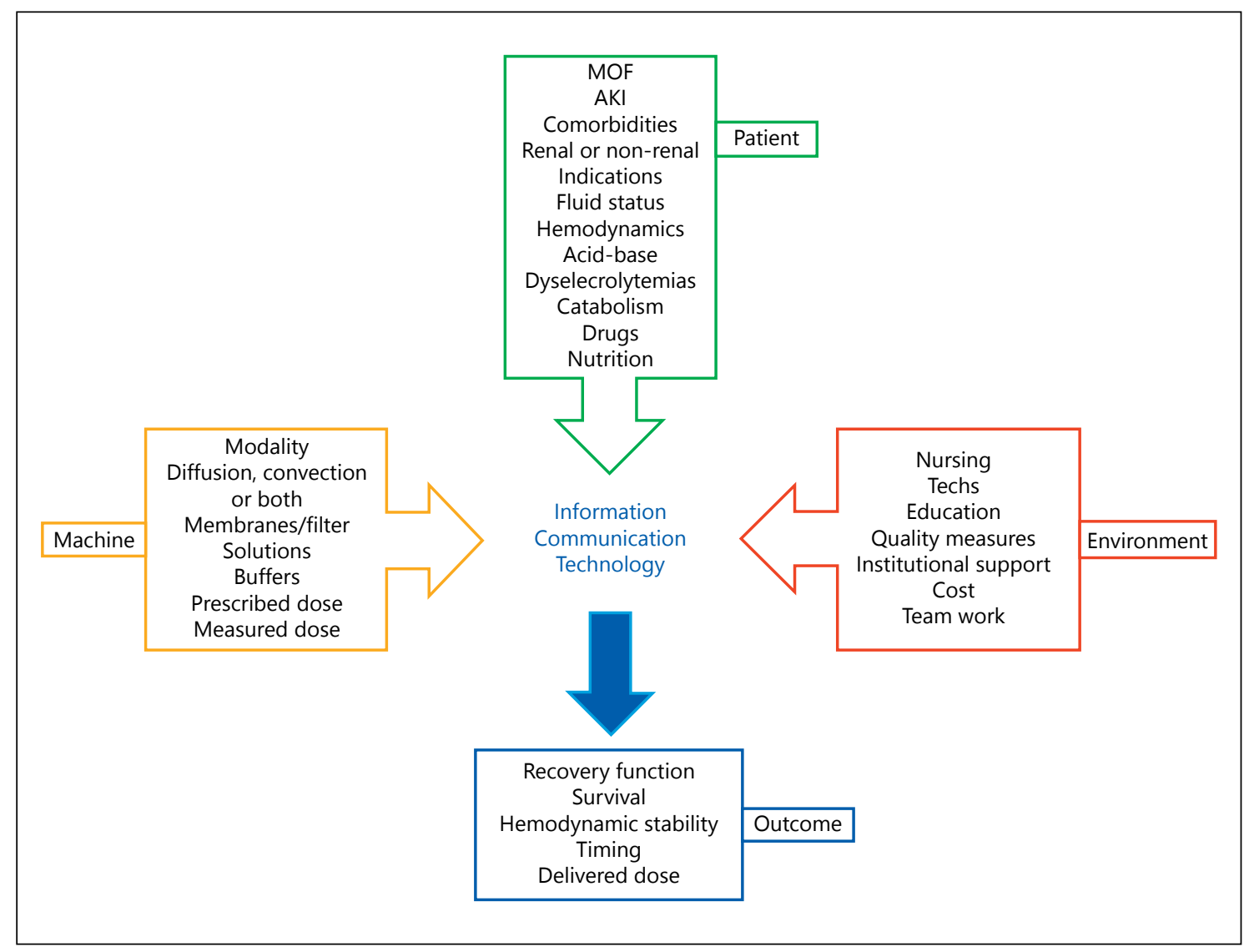

Fig. 3. Determinants allowing adequate RRT prescription and delivery. Reprinted with permission from www. ADQI.org.

feasibility influences and often determines whether the delivery of effective CRRT is possible. Availability of equipment, personnel training, environmental and staffing factors will have a role in determining whether the therapy targets established during the prescription phase are achieved.

Prompt availability of CRRT hardware, disposables and solutions is the key to achieve the delivery of an effective and timely CRRT treatment that matches the prescription as much as possible. Delay in CRRT initiation and modification in prescription due to unavailable equipment should be minimized. The group identified an ideal time window of $3 \mathrm{~h}$ between prescription and implementation of treatment.

Excellent staff training on equipment and procedures necessary to deliver the CRRT treatment must be encouraged and implemented. Indeed, appropriate use of available equipment and a deep knowledge of all the treatment phases (such as priming, bag change procedure, recirculation or blood return and disconnection) optimize ini- tiation time, decrease downtime and reduce the probability of patient complications during CRRT delivery.

Environmental and staffing issues might influence the delivery of CRRT. The environment in which AKI patients undergo CRRT highly influences the feasibility and outcomes of treatment and should be considered for an adequate prescription, delivery and monitoring of this therapy. In particular, the technical and non-technical skills of the staff involved in the management of CRRT, as well as the downtime related to the institution and patient needs (e.g., radiological evaluation), should all be considered during treatment phases. The availability of dedicated structures and qualified staff (e.g., nurses or technicians) is essential for success.

Question 5: Can technology assist in the process of prescription and dynamic re-evaluation of physiological therapy targets?

- Several technological tools can be used to monitor target achievement and to suggest modifications in prescription (Grade C). 
Table 2. Components for CRRT prescription and delivery. Terminology refers to published nomenclature [15-19]

\begin{tabular}{|c|c|}
\hline Elements & Statement \\
\hline Techniques & $\begin{array}{l}\text { Technique (SCUF, CVVH, CVVHD, CVVHDF) must be chosen according to patient needs (targets), } \\
\text { resources and hardware availability, setting compatibility (vascular access, membrane, blood flow, } \\
\text { anticoagulation, filtration fraction) and expertise }\end{array}$ \\
\hline Vascular access & Vascular access choice and management should follow KDIGO guidelines \\
\hline Equipment & $\begin{array}{l}\text { We suggest to use integrated dedicated machines (CRRT) with state of the art built-in safety functionality } \\
\text { according to availability and expertise. Specific pediatric equipment should be preferred for patients below } \\
15 \mathrm{~kg} \text { of body weight }\end{array}$ \\
\hline Anticoagulation & $\begin{array}{l}\text { Anticoagulation according KDIGO guidelines and local practice. Anticoagulation should ensure optimal } \\
\text { delivery without compromising patient safety. Anticoagulation should not be escalated to correct wrong } \\
\text { settings, failing access or to extend filter life beyond label recommendations }\end{array}$ \\
\hline Solutions & $\begin{array}{l}\text { Dialysate and replacement fluids should be appropriate to clinical targets, treatment modality prescription } \\
\text { and anticoagulation }\end{array}$ \\
\hline Settings & $\begin{array}{l}\text { Settings should consider patient characteristics/needs, hardware and technique selected [15-19]. Operational } \\
\text { characteristics should be planned in order to maximize filter patency, membrane performance and } \\
\text { achievement of targets }\end{array}$ \\
\hline Monitoring & $\begin{array}{l}\text { Monitoring should be done frequently or continuously through connectivity tools. Integrated data collection } \\
\text { (patient and machine) is strongly recommended }\end{array}$ \\
\hline Other techniques & $\begin{array}{l}\text { CRRT can be done in conjunction or sequentially with hemoperfusion, CPFA, ECCOR, liver support, ECMO } \\
\text { or other organ support techniques in case of specific indications [15-19] }\end{array}$ \\
\hline
\end{tabular}

- Manual, authorized or automatic feedback technology is today available in chronic dialysis machines and it should be promoted in CRRT machines of future generation (Grade $\mathrm{E}$ ).

- We strongly recommend integration of patient and machine signals through IT tools and connectivity with EMR and data collection systems (Grade B).

- Data should be used for QA and CQI purposes in the center, and also for registries or possible pragmatic trials (Grade B).

Currently available CRRT machines have advanced software, which enables the safe and correct provision of a fluid balance target and correction when error is detected, administration and management of citrate anticoagulation, temporary blood flow reduction when excess negative access pressures are detected, maintenance of filtration fraction with a given blood flow and fluid settings and inclusive on-screen live tutorial tools [26, 27]. These software tools may function automatically or prompt the user with alarms to modify the settings (fig. 4).

Role of Technology in CRRT for the Management of Critically Ill Patients
These tools will make it more likely that CRRT will operate with increased safety and more efficiently without interruptions or avoidable down time $[28,29]$ (Level V; Grade E). When the technology is available and used correctly, recent advances may also prevent premature terminal clotting events (e.g., filter life less than $4 \mathrm{~h}$ ). This permits better dose delivery [30] (Level $\mathrm{V})$, less cost and waste. Software design and technology also assists users to reduce the need for constant attention and troubleshooting, and therefore saves nursing time. More formal evaluation for these proposed benefits should be an important part of the research agenda.

Manual, authorized or automatic feedback technology is today available but varies across CRRT machines. Manufacturer branding or trademarking of these options provides an important market edge and a sales advantage (Level V). However, as advances in safety become widespread, some of these options will be present in all machines. Regulatory authorities may mandate some of 
Fig. 4. The role of prescription-delivery feedback loop during CRRT. Includes solutes, acid-base, fluids and other patient and treatment variables. a According to the actual patient's physiological requirement (green line), the clinician sets the CRRT prescription (purple line) over time. Depending on several factors, such as patient, treatment and environment, the prescription will determine a specific treatment delivery (red line). Although the prescription is continuously adapted (points I, II, III, IV, etc.), a marked difference is evident between the actual patient's requirements and the prescription. $\mathbf{b}$ If a prescription delivery feedback loop is used (e.g., biofeedback), the differences between the treatment delivery and patient's physiologic requirement might be instantaneously measured (in this example, through the angle between the 2 curves). In this setting, the delivery may be modulated according to the patient's needs, through automated, assisted or manual changes of the prescription (points 1, 2, 3, 4, etc.). c As continuous changes are made according to the feedback analysis, the time average deviation between the delivery and the patient's need (area A, B, C, D, etc.) is progressively reduced over time. Furthermore, as the maximal deviation between the delivery and the patient's need progressively decreases during the treatment, increasingly small variations are required to actually delivery an 'adequate treatment' (the overlap between the green and the red curves). Reprinted with permission from www.ADQI.org.
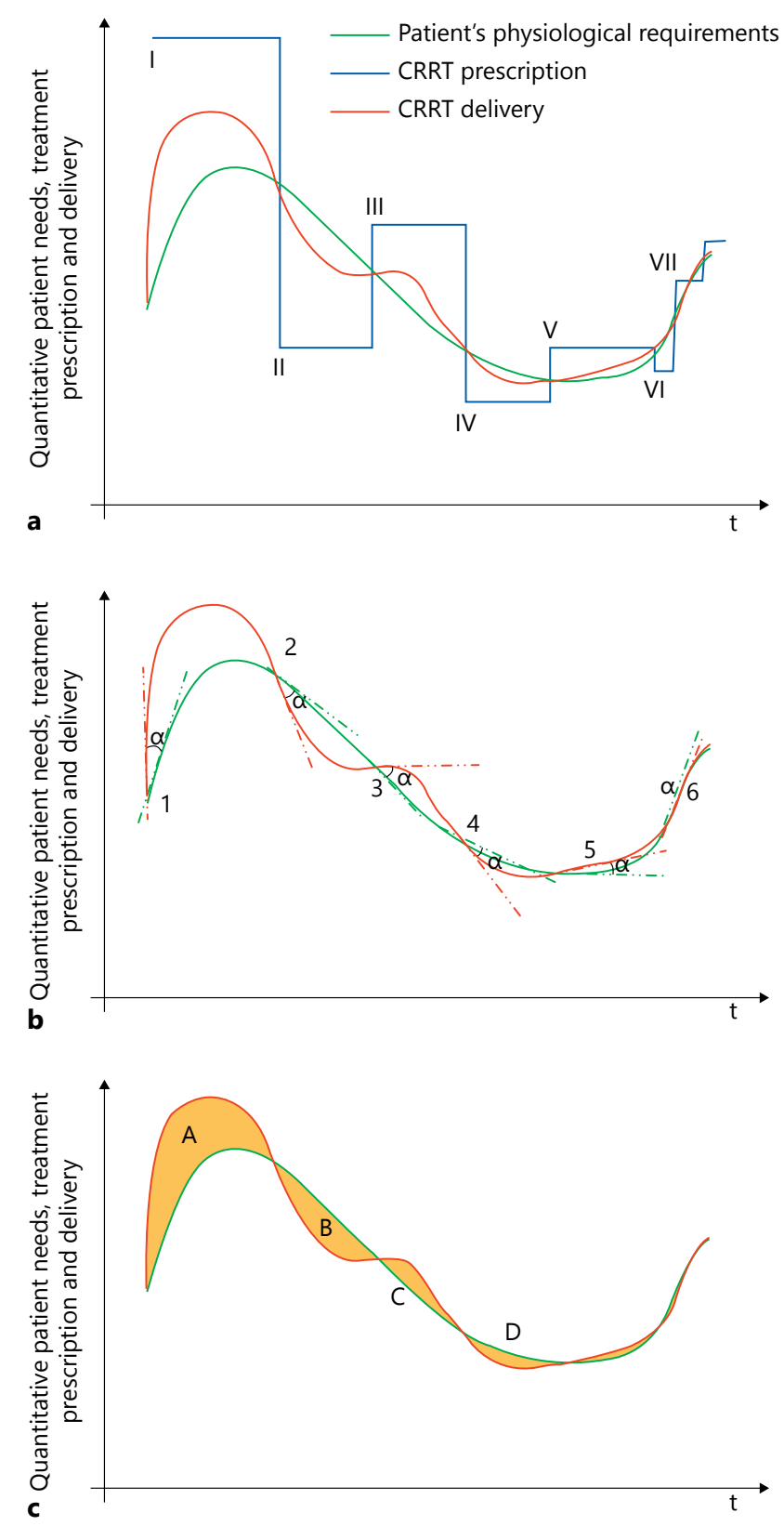

these protections (such as positive or fluid balance error detection, alarms and automated correction) whenever these errors are associated with increased morbidity and mortality [31-33] (Level V, Grade E).

Numerous data can be electronically transmitted either during or following completion of a CRRT treatment. This approach is currently more advanced in chronic dialysis programs. In the acute setting, quality indicators of CRRT should include data on patient demographics, CRRT indication, treatment prescription and modality used, vascular access and membrane pressures during treatment, target achievement of fluid balance, prescribed and delivered dose $[30,34,35]$ (Level V), solute and acid-base control, alarm events and errors, user comments and reports relevant to any special measure required for patient precision care. 
These data may be added to establish clinical information systems that, with appropriate data processing, can provide meaningful reports for clinicians and managers involved in the treatment of AKI (Level E). The power of such reports may be made real when funding and additional resources for AKI support become necessary. Undoubtedly, CRRT safety and a quality continuum will benefit from such enriched data [30] (Level V, Grade E).

Data should not only be used for QA and CQI purposes in each center but should also be available for multicentric registries and pragmatic trials. An advanced, comprehensive quality program relies on a rich and reliable data source. Data can be analyzed locally to assist clinicians in their daily work to contrast each center results against published reports and can be offered to group national or international registries when available [36] (Level IV). The contribution to, and affiliation with, such registries will enable meaningful collaborative and multicentric trials.

Question 6: How can adequacy of treatment and dose delivery be described and technically supported?

- The measurement of dose delivery is usually based on urea kinetics. Effective dose delivery should be measured at least daily. Dose parameters should be characterized as described in recently harmonized nomenclature (Grade A) [15-19].

- When utilizing a marker molecule such as urea, dose prescription and delivery can be expressed as clearance $(\mathrm{ml} / \mathrm{kg} / \mathrm{h})$ or as a dimensionless Kt/V (fractional clearance), which in case of continuous therapies equals the standard $\mathrm{Kt} / \mathrm{V}$ (StKt/V) of intermittent therapies and reflects solute removal.

- Downtimes negatively affect dose delivery. Fluid overload may negatively affect effective dose delivery since real volume of solute distribution in the body is underestimated if ideal body weight is used as a reference for prescription. Nomograms reported in figure 5 can help clinicians evaluate the expected or effective Kt/V results when treatment is prescribed in milliliters per kilogram per hour (assuming 24-hour treatment and ideal body weight).

- The concept of adequacy of dialysis is broader than urea $\mathrm{Kt} / \mathrm{V}$ or related parameters; it must also include residual renal function at different AKI stages (worsening and recovering) and multiple other parameters including, importantly, fluid management and nutrition (Grade A; fig. 6).

Prescription and measurement of dose delivery in dialysis and also in CRRT is generally made using urea as a marker solute. Urea does not carry specific toxicity but it is strictly related to protein metabolism and while its accumulation describes dialysis requirements, its removal describes dialysis efficiency. One of the important functions of the native and the artificial kidney is to remove urea accumulated in the body. The concept of 'clearance' has been introduced to measure the removal of solutes independently of solute blood levels and to make comparisons between disparate therapies and native kidneys possible.

While blood urea levels vary exponentially in intermittent hemodialysis during treatment, levels remain fairly constant over time in CRRT blood, and thus, the correlation between daily clearance and urea removal is approximately linear. The same is true for the correlation between daily fractional clearance (normalized for solute volume of distribution $=\mathrm{Kt} / \mathrm{V}$, or normalized for body weight $=\mathrm{ml} / \mathrm{kg} / \mathrm{h}$ ) and solute removal. This explains why $\mathrm{Kt} / \mathrm{V}$ and StKt/V are identical when treatment lasts $24 \mathrm{~h}$ and solute blood levels remain constant.

Differences between prescription and effective delivery may occur when fluid overload is present but treatment is prescribed using ideal body weight (fig. 5). The same is true when treatment is prescribed for $24 \mathrm{~h}$ but significant downtime occurs (fig. 5, lower panel). When prescribing and measuring the dose of CRRT, one can use clearance (as $\mathrm{ml} / \mathrm{kg} / \mathrm{h}$ ) or $\mathrm{Kt} / \mathrm{V}$ (in continuous therapies, equivalent to the $\mathrm{StKt} / \mathrm{V}$ of intermittent hemodialysis), but one must be aware of potential differences between prescribed and delivered treatment dose'.

Today, some CRRT machines are capable of measuring and displaying current efficiency (clearance or Kt/V) and project values over time, based on current parameters. In case of downtime or treatment malfunction, these machines modify the projected values and - in some cases - they display an automatic feedback message designed to compensate for the downtime. In other machines, new software has been designed to suggest corrective measures to achieve the desired results. It is for these reasons that the terminology concerning dose must be harmonized according to recommended standards [15-19].

Finally, the use of one single marker molecule to define treatment adequacy has been questioned. There is consensus that 'adequacy' should be a broader term that includes several aspects of the treatment. Because the final targets of treatment can be volume control, metabolic correction, electrolyte re-equilibration, acid-base homeostasis, cardiovascular stability and organ protection among others, there is general consensus that a multidimensional approach must be considered to achieve adequacy of treatment (fig. 6). If a treatment achieves ade- 
Fig. 5. Nomogram to achieve precision delivery of CRRT when dose is prescribed according to ideal weight and fluid overload or downtime may induce variations in effective delivery. Reprinted with permission from www.ADQI.org.

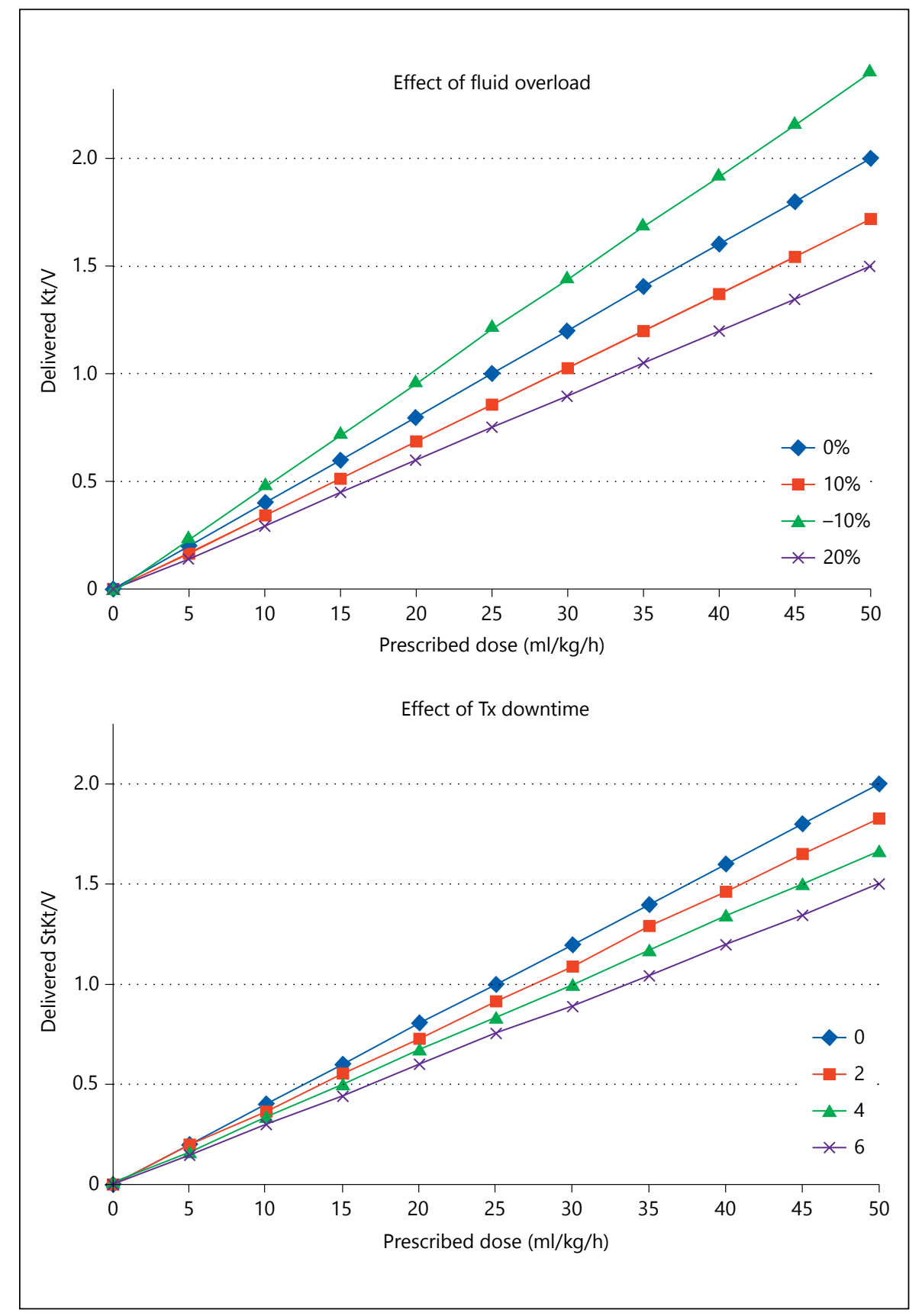

quate $\mathrm{Kt} / \mathrm{V}$ or a perfect correction of acidosis but fluid overload remains unchanged or severe hypotension occurs, the final judgment should be that adequacy of treatment has not been achieved. Only in presence of results that satisfy multiple parameters (fig. 6, lower right panel), treatment can be considered adequate. Still, adequate is not a synonymous for optimal; technology can definitely help to further improve each single arm of the efficiency/ tolerance domain map.
Question 7: When should 'other' techniques be prescribed?

- In spite of little or no evidence for specific extracorporeal therapies in sepsis, there is a common agreement that precision therapy (personalized) and single-patient approach should be considered whenever possible, based on the theoretical advantages of specific techniques and the rationale for their application (Grade E). 


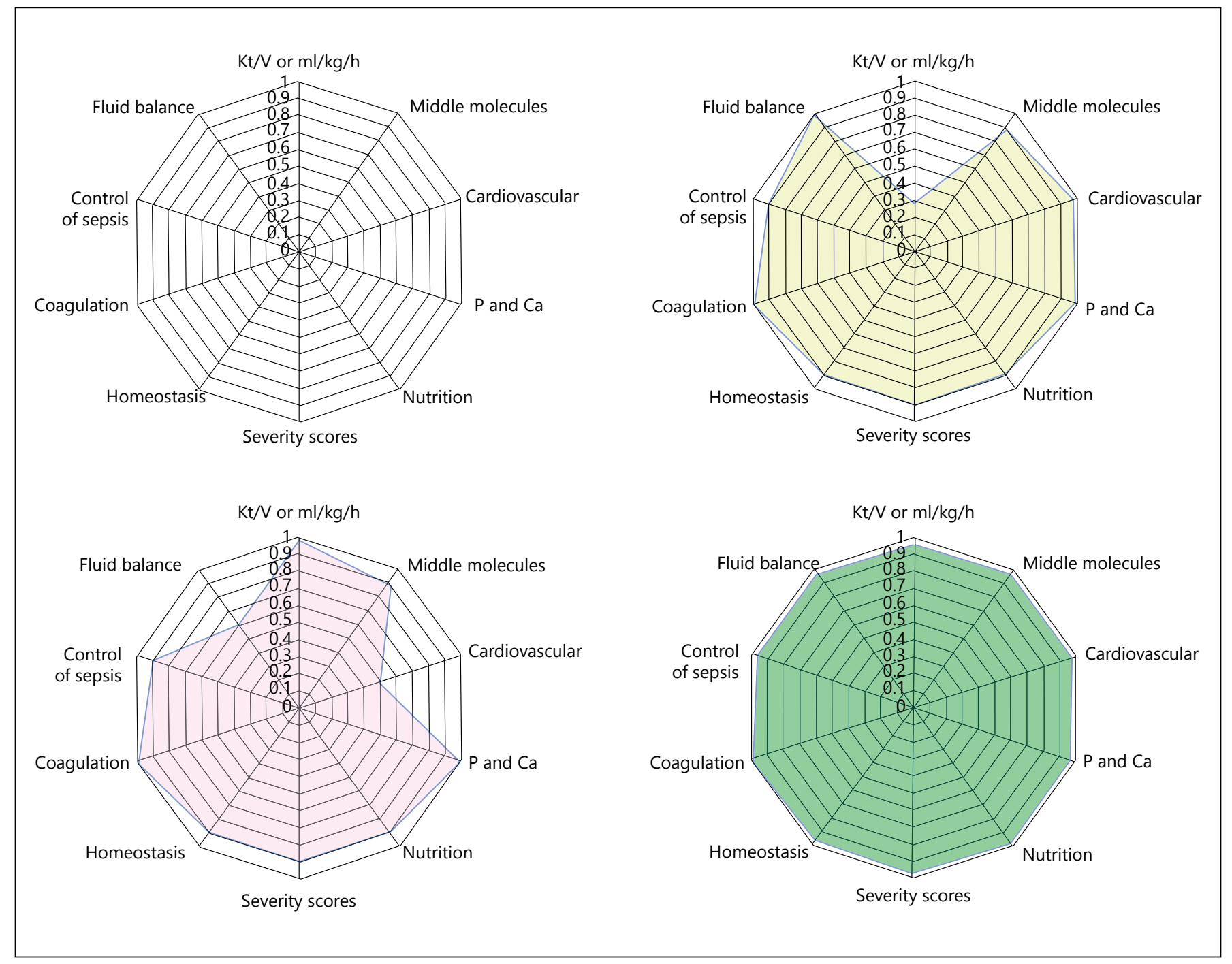

Fig. 6. Radar plot describing the broader spectrum of adequacy. Every single aspect can be measured in arbitrary or objective units, but the final result of the polygon in the radar plot will describe a comprehensive evaluation of treatment adequacy. Some examples in plots (top right and bottom left) describe incomplete adequacy while in the bottom right panel a fully adequate treatment is described. Reprinted with permission from www.ADQI.org.
- High-volume hemofiltration (HVHF) is not recommended for blood purification in sepsis. However, higher hemofiltration doses may be prescribed to match individual patient needs (Grade 1A).

- Polymyxin B (PMX B) hemoperfusion is not recommended for the general population but it should be considered in patients post surgery for abdominal sepsis and septic shock (Grade C).

- Plasma exchange, liver support, ECCOR and CRRT/ ECMO should be used only for specific indications based on center and physician expertise and results of ongoing trials (Grade D).

Role of Technology in CRRT for the Management of Critically Ill Patients
- In order to maximize precision CRRT, equipment designed specifically for children should be used for patients below $15 \mathrm{~kg}$ of body weight and especially in neonates (Grade A).

- Today, CRRT technology should be seen more like a complex and integrated system that provides multiple organ support therapy (MOST), offering the possibility to substitute or support the function of several organs via extracorporeal circuits with specific characteristics.

HVHF was initially proposed as an adjunctive treatment of septic shock complicated by AKI. Prospective in- 
terventional studies showed an early hemodynamic benefit, faster weaning from inotropic support, lessened organ failures, and reduced intensive care unit length of stay $[37,38]$. A survival benefit was suggested $[37,38]$ but not confirmed by prospective randomized controlled studies $[39,40]$. The 2015 Vicenza Nomenclature Standardization Initiative (NSI) defined HVHF as a continuous convective treatment with a (prescribed) target dose greater than $35 \mathrm{ml} / \mathrm{kg} / \mathrm{h} \mathrm{[15-19].} \mathrm{A} \mathrm{dose} \mathrm{exceeding} 45 \mathrm{ml} / \mathrm{kg} / \mathrm{h}$ represents very HVHF (VHVHF) [15-19]. Intermittent procedures using brief VHVHF episodes (100-120 ml/ $\mathrm{kg} / \mathrm{h}$ for $4-8 \mathrm{~h}$ ), followed by conventional $\mathrm{CVVH}$, are identified as 'pulse' HVHF [15-19, 38]. The high-volume intensive care (IVOIRE) trial [39] randomized 140 septic shock patients with AKI to receive a CVVH dose of either 35 or $70 \mathrm{ml} / \mathrm{kg} / \mathrm{h}$ for 4 consecutive days. Mortality at 28 and 90 days was comparable between groups. Renal recovery was high with less than $5 \%$ of patients remaining dialysis-dependent at 3 months. A recent meta-analysis found no effect of HVHF on sepsis mortality [41]. Zhang et al. [40] compared $50 \mathrm{ml} / \mathrm{kg} / \mathrm{h}$ with $85 \mathrm{ml} / \mathrm{kg} / \mathrm{h}$ dose in 280 patients with severe sepsis (half of them with shock) and AKI. Mortality at 28, 60 and 90 days was similar between groups. Routine use of HVHF in critically ill patients with severe sepsis and/or septic shock cannot be recommended except in the setting of a randomized clinical trial (Grade 1A).

The PMX B hemoperfusion technique employs a cartridge containing polystyrene fibers covered by PMX B that specifically bind circulating endotoxin. The treatment can be performed even in the absence of CRRT. A meta-analysis demonstrated beneficial hemodynamic effects of PMX B treatment in septic shock [42]. The Early Use of Polymyxin B Hemoperfusion in Abdominal Sepsis trial included patients exposed to high circulating endotoxin levels within the scope of abdominal septic shock [43]. Sixty-four patients were randomly assigned to receive either standard treatment or standard treatment plus 2 sessions of PMX B therapy. The PMX B group had better hemodynamic parameters, faster resolving of organ failure and lower 28-day mortality [43]. Payen et al. [44] applied a similar study protocol to 243 patients with peritonitis-induced septic shock. The PMX B group had no improvement in organ failure and a non-significant higher mortality rate [44]. However, PMX B treatment was incomplete in $38 \%$ of patients which was higher than previously reported [44]. Awaiting further studies, PMX $B$ hemoperfusion is currently not recommended but can be applied to patients with abdominal sepsis and septic shock (Grade C).
Isolated or continuous ultrafiltration should be provided to patients with congestive heart failure to remove fluid overload when response to diuretics is insufficient or absent. In spite of scanty evidence for specific longterm benefits, the trials have demonstrated superiority in symptoms relief and fluid balance control compared to diuretics alone in refractory patients.

Coupled plasmafiltration adsorption should be explored as a potential treatment for sepsis and rhabdomyolysis due to its capacity of removing protein-bound solutes and large molecular weight solutes without requiring external fresh plasma reinfusion. The rationale in sepsis is the reconstitution of a certain immuno-homeostasis due to non-selective pro- and anti-inflammatory mediators' removal.

High cutoff membranes as well as functionalized membranes should be studied in AKI and sepsis due to their enhanced capacity of solute removal. Antibiotic and protein losses should be carefully evaluated.

Liver support should be further evaluated combining plasma exchange, plasmafiltration-adsorption, direct hemoperfusion and other techniques designed to remove liver failure-derived retention molecules.

In conclusion, today's CRRT machines should be seen as platforms for most, rather than simple RRT tools. CRRT technology should be seen as a multi-component, complex and integrated system designed to substitute or support several organ functions by means of extracorporeal circuits with specific characteristics.

Question 8: What are the main areas for future research in CRRT technology?

- High cutoff membranes (HCO);

- Sorbents;

- CRRT combined with ECMO or $\mathrm{ECCO}^{2} \mathrm{R}$;

- Functionalized membranes;

- Automatic biofeedback;

- Electronic support to decision process.

\section{High Cut-Off Membranes}

A 'high cutoff' is coined as a membrane with a cutoff equal to $60 \mathrm{kDa}$. In the NSI, it was described as membranes with a cutoff value that approximates the molecular weight of albumin [15-19]. A pilot trial in septic patients with AKI randomized 30 patients to treatment with either an HCO membrane $(60 \mathrm{kD} / \mathrm{P} 2 \mathrm{SH}$ Gambro) or a classic membrane (35 kD/Polyflux 11S) [45]. The use of the HCO membrane resulted in a significant reduction of vasopressor use and a 10-fold increase in clearance of interleukin (IL)-6 and IL1-receptor antagonist [45]. The recently completed high cutoff sepsis 
study that randomized 120 patients with septic shock and AKI to treatment with CVVHD using either a conventional or an $\mathrm{HCO}$ membrane yielded encouraging results for safety (no albumin loss) but no improvement in efficacy [46]. The study was stopped prematurely after enrolment of only 81 patients due to a lack of difference in 28 -day mortality ( $31 \%$ HCO vs. $33 \%$ conventional membrane). No difference was observed in vasopressor need, duration of mechanical ventilation or length of ICU stay [46]. To date, HCO membranes cannot be recommended for routine clinical practice. Their use should be restricted to research protocols in sepsis and rhabdomyolysis.

\section{Sorbents}

Albeit structurally different, CytoSorb and related sorbents do express excellent adsorption rates for most relevant inflammatory cytokines [47]. Cytokine adsorbing columns have a huge surface (up to $8,500 \mathrm{~m}^{2}$ ) as compared with classic CRRT membranes [47]. CytoSorb swiftly cleared $50-80 \%$ of circulating cytokines in septic animals [48], which exceeded by far the filtering potential of HCO or high-adsorptive membranes. One clinical study randomized 43 patients to standard treatment with or without CytoSorb, 6 h daily for 7 days [49]. CytoSorb treatment was associated with cytokine reduction and significantly lowered 28-day mortality. A major disadvantage of CytoSorb is its inability to adsorb endotoxin $[48,49]$. Based on current evidence, CytoSorb and other sorbents are not ready to enter clinical practice. Their potential use requires further investigation, particularly in severe inflammatory disease.

\section{CRRT Combined with ECMO or ECCO ${ }^{2} R$}

CRRT + ECMO

ECMO patients frequently develop AKI, which requires initiation of CRRT in addition to heart-lung support [50]. Utilizing the large bore and high flow ECMO cannulas seems convenient to connect both extracorporeal circuits. However, creating a 2-in-1 ECMO/CRRT system has practical consequences as well as limitations and drawbacks. To avoid air trapping in the ECMO membrane, connecting the outflow CRRT line after the ECMO pump and oxygenator [51] is safer, but is hampered by the existing positive pressure in this position. To make the connection possible, it is usually necessary that CRRT alarms be modified to tolerate positive return pressures, where negative readings are normally present.

As a high-flow system equipped with heparin-coated membranes and circuits, ECMO requires no or only min- imal additional anticoagulation to ensure circuit patency [50]. In contrast, CRRT is a low-flow system that demands specific anticoagulation to avoid early circuit clotting [51]. Embedding a full CRRT device serially with an ECMO circuit may obviate these shortcomings. However, the dramatic difference in flow and pressure will increase shear stress, which exposes patients to disseminated intravascular coagulation [51]. For these reasons (and based on limited data), we strongly argue against the use of CRRT in series within an ECMO circuit; parallel circuit designs avoid such problems. Randomized trials comparing both systems should be performed in the near future in order to allow recommendation of the best clinical practice approach.

\section{$\mathrm{CRRT}+\mathrm{ECCO}^{2} \mathrm{R}$}

As for CRRT, $\mathrm{AV}-\mathrm{ECCO}^{2} \mathrm{R}$ can be adequately performed under regional citrate anticoagulation $[51,52]$. Because AV-ECCO ${ }^{2} \mathrm{R}$ blood flow is relatively limited (up to $11 / \mathrm{min}$ ), the risk of shear stress produced by embedding CRRT inside an AV-ECCO ${ }^{2} \mathrm{R}$ circuit is low [51, 52].

\section{Functionalized Membranes}

Different surface and structure modifications have been introduced on new membranes to increase biocompatibility, reduce thrombogenicity and to modify sieving and adsorption properties. Surface functionalization has been also attempted as in the case of vitamin E-bound membranes, where alpha-tocopherol has been covalently bound to a polysulfone membrane to reduce oxidant species generation and oxidant stress, to prevent or treat ischemia-reperfusion injury and to improve the inflammatory pattern in sepsis [53]. These membranes should be further investigated.

\section{Automatic Biofeedback}

The nature of the critically ill patient requires a continuous control of CRRT delivery and strict adherence to prescription, as well as maximization of patient tolerance. The treatment should avoid sudden and sharp variations of physiological parameters, and allow slow and precise correction of fluid, electrolyte and metabolic imbalances. To prevent complications in online intra-dialytic monitoring, the systems currently used in chronic dialysis continuously measure various hemodynamic and biochemical parameters. Such online monitoring systems are particularly useful in short intermittent dialysis techniques, where the risk of 'un-physiology' is greater due to the high efficiency of the treatment. 
Fig. 7. Different options for a CRRT feedback. Manual, authorized, automatic. Reprinted with permission from www.ADQI. org.

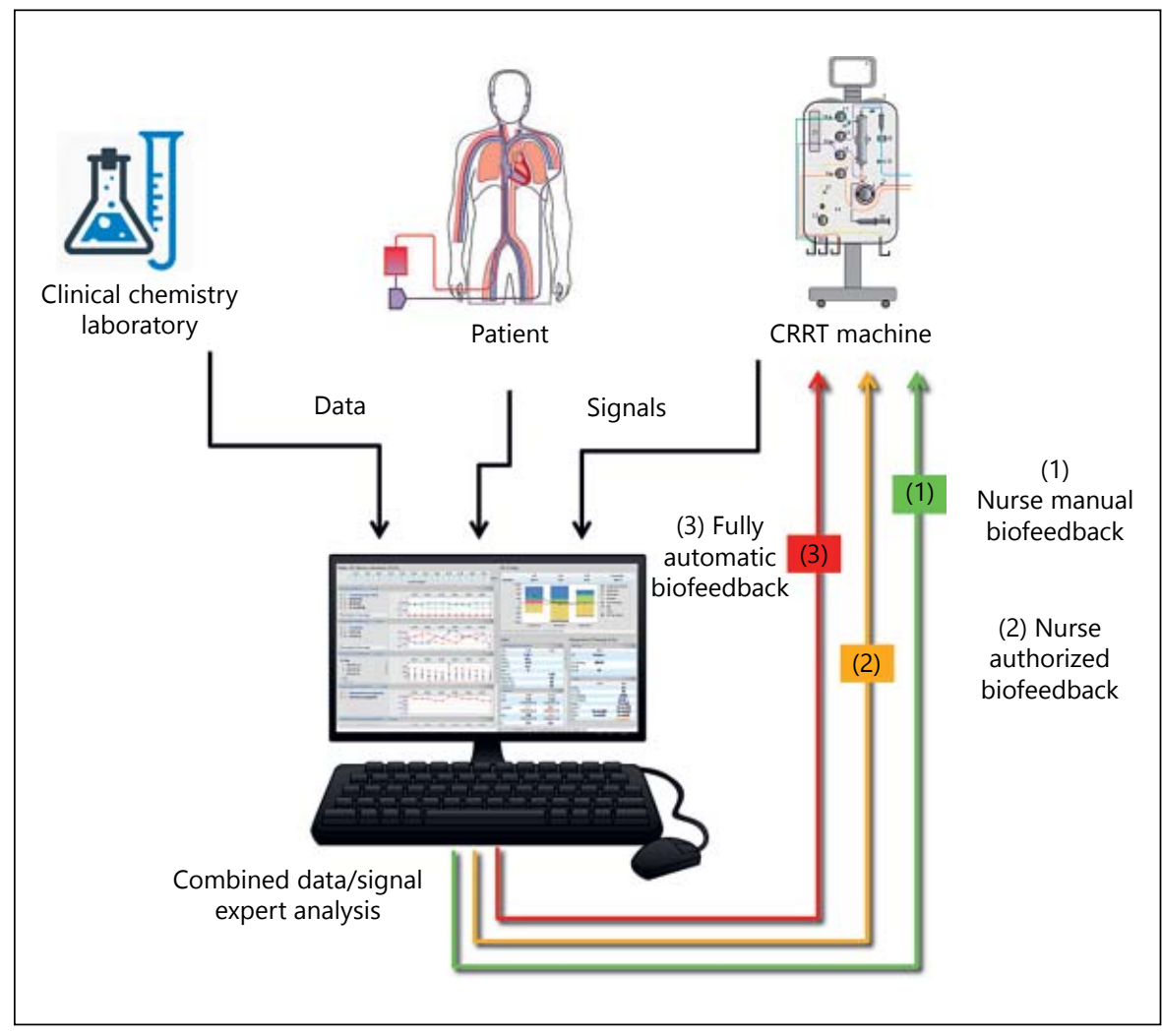

The development of such systems is based on a 3-step analysis that can be summarized as follows: (1) each patient is different (this requires precision CRRT and personalized prescription); (2) patient characteristics vary during treatment (this requires that dynamic prescription also changes over time). A solution to this problem has been attempted with the use of pre-set profiles of ultrafiltration and dialysate composition, but instead of being based on actual signals from the patient, the profiles are blind to the patient needs and outcomes; and (3) patient and machine signals, describing actual clinical and technical conditions, should be used to drive the delivery of therapy and to reassess prescription (today, this is possible through multi-inputmulti-output controllers and actuators, which constitute the basis of a 'smart' biofeedback; fig. 7).

In CRRT, patient and machine data collection should also feed into EMRs. Data should be used immediately to fulfill specific objectives:

- Achievement of an adequate ultrafiltration rate and profile over time, optimizing fluid balance (with minimal deviations from prescribed values) and cardiovascular response to fluid withdrawal while ensuring maximal hemodynamic stability. This is possible through integration of bioimpedance and online he- matocrit measurements resulting in important input on overall patient fluid status and actual circulating blood volume changes. Maintenance of hemodynamic stability and smooth operation of the CRRT machines with minimal interruptions will result in a more effective delivery of the treatment prescribed.

- Adequate dose delivery with minimal deviations from prescribed values. This can be achieved in different ways depending on hardware and software integration.

- Thermal and energy balance with biofeedback-driven temperature control. This feedback can be designed to achieve a specific energy balance $(\mathrm{kJ} / \mathrm{h})$ or a target temperature control by adjusting dialysate or replacement fluid temperature according to signals coming from temperature sensors placed on blood and dialysate lines. It should be remembered that a significant heat loss can occur when the extracorporeal circuit is exposed to room temperature.

- Circuit pressure control. This automatic feedback should provide the best blood flow adjustment to pressure variations measured within the circuit. This would help clinicians to deal with a malfunctioning catheter and provide early warning of access malfunc- 
Fig. 8. Different options for data collection in CRRT. Reprinted with permission from www.ADQI.org.

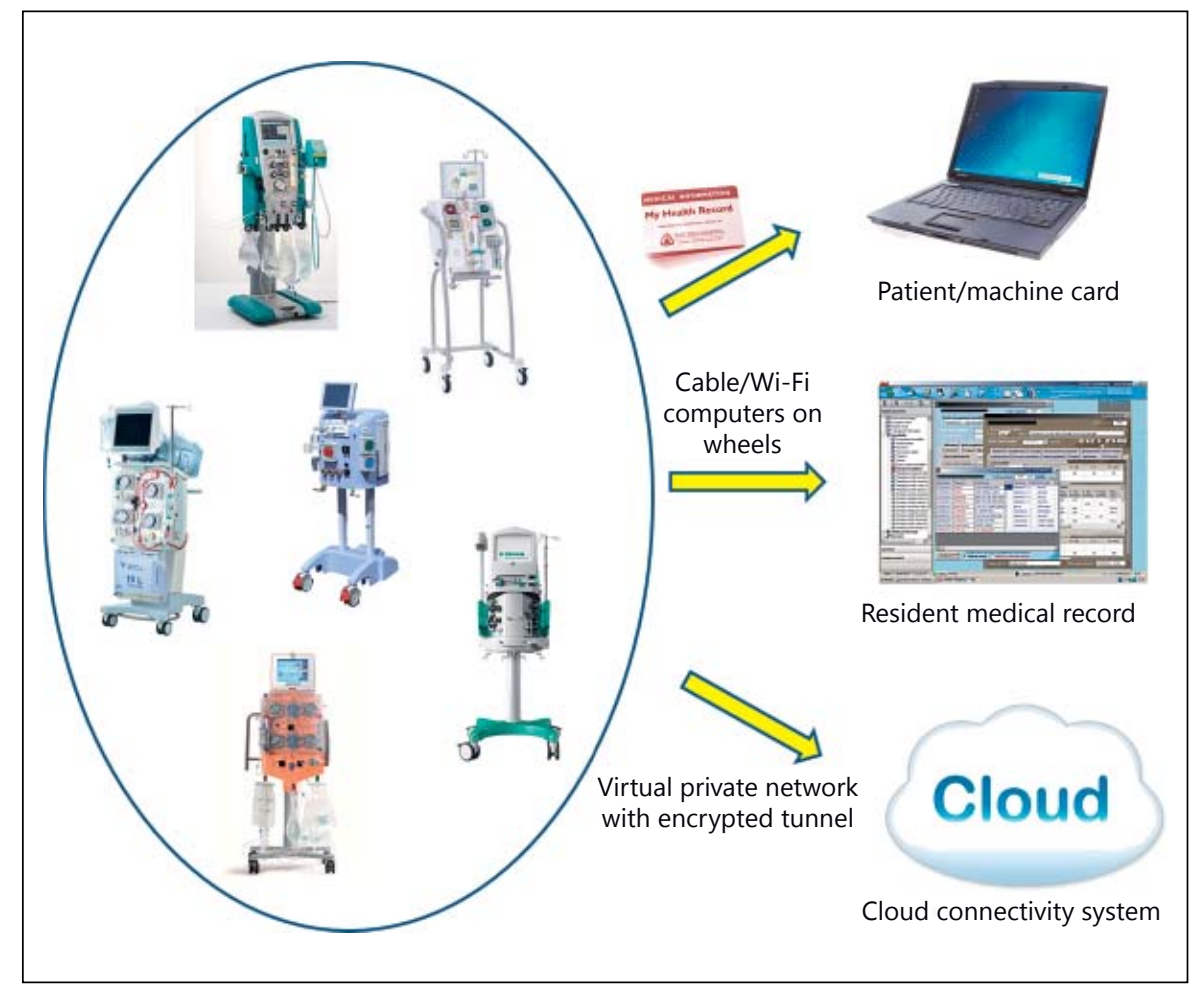

tion, a very common cause of inappropriate treatment delivery.

- Acid-base and electrolyte control could be achieved via a biochemical feedback based on online chemical sensors and actuators operating on a variable concentration of dialysate and replacement solution or modification of flow rate of the solutions.

The use of currently available measurement devices is strongly recommended, as well as the development of new 'sensors' for continuous monitoring during the CRRT session. Such devices should be simple, non-invasive and cheap, possibly integrated with the machine, sterile and biocompatible if they come in direct contact with blood, integrated with an external EMR and potentially linked to automatic actuators and, lastly, low cost.

\section{CRRT Connectivity}

All the data that are continuously generated by the patient and machine during treatment must be collected. While automatic feeding of patient data into the EMR is important for clinical purposes, data collection from CRRT machines is crucial both for technical and clinical purposes.

Machine connectivity can be provided via different tools. Machine and patient chip cards can be used to ex- tract data from single treatments from the front-end terminal (CRRT machine). Cable or wireless connectivity may permit the download of technical and clinical parameters from single or multiple machines and to analyze single treatment data as well as trends or statistics in multiple treatments. Cloud-based connectivity could help clinicians to generate virtual registries and analyze single treatments or center performance in absolute terms or relative to other units. This may result in important feedback to clinicians, to either strictly control outliers or to change policies and procedures in case of repeatedly unsatisfactory results (fig. 8).

\section{Electronic Support to the Decision Process}

Data collected and stored in EMRs may be rapidly evaluated and managed by ad-hoc designed electronic sniffers, which may alert clinicians about dangerous trends or unwanted effects of CRRT. Solutions to the problem may be listed as suggestions or even automatically fed back into devices such as pumps and CRRT machines. The feedback may either require manual application of the necessary change by a nurse/physician, authorization of an operational change proposed by the system or operate automatically. These procedures will require thorough validation. 


\section{Conclusions}

Since the first ADQI Meeting in New York 16 years ago, the use of CRRT for the management of AKI in critically ill patients has greatly benefited from important technological advances in machine design, an improved understanding of the timing and dose of RRT and the application of novel, more complex modalities. Such improvements have greatly expanded the field of extracorporeal therapies in the ICU. Concurrently, the treatment of the AKI patient has evolved from the single replacement of kidney function to the support of the patient as a whole, incorporating technologies that assist in the management of multiple organ dysfunctions. Necessarily, such integrated care must be supported by information technologies, which assist in data collection and integration, delivery of a personalized prescription and measurement of results. Such technologies allow continuous feedback adjustment of each individual treatment, and permit ongoing quality improvement of the program as a whole. We expect that our recommendations for newer technological applications and research will continue to evolve into prescription and delivery of precision CRRT, leading to improved patient survival, minimization of complications and enhanced functional recovery.

\section{Disclosure Statement}

None.

\section{References}

1 Ricci Z, Bonello M, Salvatori G, Ratanarat R, Brendolan A, Dan M, et al: Continuous renal replacement technology: from adaptive devices to flexible multipurpose machines. Crit Care Resusc 2004;6:180-187.

2 Cerdá J, Ronco C: Modalities of continuous renal replacement therapy: technical and clinical considerations. Semin Dial 2009;22:114122.

3 Cruz D, Bobek I, Lentini P, Soni S, Chionh $\mathrm{CY}$, Ronco C: Machines for continuous renal replacement therapy. Semin Dial 2009;22: 123-132.

4 Ricci Z, Ronco C: Technical advances in renal replacement therapy. Semin Dial 2011;24: 138-141.

5 Ronco C, Kellum JA, Mehta R: The acute dialysis quality initiative: the New York conference. Adv Ren Replace Ther 2002;9:248-251.

6 http://www.adqi.org/. 2016.

7 Murray P, Mehta RL, Shaw A, Ronco C, Endre Z, Kellum JA, et al: Potential use of biomarkers in acute kidney injury: report and summary of recommendations from the 10th acute dialysis quality initiative consensus conference. Kidney Int 2014;85:513-521.

8 McCullough PA, Kellum JA, Haase M, Müller C, Damman K, Murray PT, et al: Pathophysiology of the cardiorenal syndromes: executive summary from the eleventh consensus conference of the acute dialysis quality initiative (ADQI). Contrib Nephrol 2013; 182:82-98.

9 Villa G, Ricci Z, Romagnoli S, Ronco C: Multidimensional approach to adequacy of renal replacement therapy in acute kidney injury. Contrib Nephrol 2016;187:94-105.

10 Ronco C, Kellum JA, Haase M: Subclinical AKI is still AKI. Crit Care 2012;16:313.

11 Zaragoza JJ, Villa G, Garzotto F, Sharma A, Lorenzin A, Ribeiro L, et al: Initiation of renal replacement therapy in the intensive care unit in Vicenza (IRRIV) score. Blood Purif 2015; 39:246-257.

12 Lachance P, Villeneuve PM, Wilson FP, Selby NM, Featherstone R, Rewa O, et al: Impact of e-alert for detection of acute kidney injury on processes of care and outcomes: protocol for a systematic review and meta-analysis. BMJ Open 2016;6:e011152.

13 Ricci Z, Romagnoli S, Villa G, Ronco C: Modality and dosing of acute renal replacement therapy. Minerva Urol Nefrol 2016;68:78-86.

14 Villa G, Ricci Z, Ronco C: Renal replacement therapy. Crit Care Clin 2015;31:839-848.

15 Ronco C: Standard nomenclature for renal replacement therapy in acute kidney injury: very much needed! Blood Purif 2014;38:I-II.

16 Neri M, Cerdà J, Garzotto F, Villa G, Ronco C: Nomenclature for renal replacement therapy in acute kidney injury; in Kellum JA, Bellomo R, and Ronco C (eds): Continuous Renal Replacement Therapy, 2016, pp 21-34.

17 Neri M, Villa G, Garzotto F, Bagshaw S, Bellomo R, Cerda J, et al: Nomenclature for renal replacement therapy in acute kidney injury: basic principles. Crit Care, in press.

18 Villa G, Neri M, Bellomo R, Cerda J, De Gaudio R, De Rosa S, et al: Nomenclature for renal replacement therapy in acute kidney injury: practical applications. Crit Care, in press.

19 Ronco C: The Charta of Vicenza. Blood Purif 2015;40:I-V.

20 Kidney Disease: Improving Global Outcomes (KDIGO) Acute Kidney Injury Work Group: KDIGO clinical practice guideline for acute kidney injury. Kidney Int Suppl 2012;2:124138.

21 Bagshaw SM, Cruz DN, Gibney RT, Ronco C: A proposed algorithm for initiation of renal replacement therapy in critically ill patients. Crit Care 2009;13:317.
22 Ostermann M, Dickie H, Barrett NA: Renal replacement therapy in critically ill patients with acute kidney injury - when to start. Nephrol Dial Transplant 2012;27:22422248.

23 Macedo E, Mehta RL: When should renal replacement therapy be initiated for acute kidney injury? 2011;24:132-137.

24 Macedo E, Mehta RL: Tailored therapy: matching the method to the patient. Blood Purif 2012;34:124-131.

25 Mehta RL: Indications for dialysis in the ICU: renal replacement vs. renal support. Blood Purif 2001;19:227-232.

26 Brain MJ, Roodenburg OS, Adams N, McCracken P, Hockings L, Musgrave S, et al: Randomised trial of software algorithm driven regional citrate anticoagulation versus heparin in continuous renal replacement therapy: the filter life in renal replacement therapy pilot trial. Crit Care Resusc 2014;16: 131-137.

27 Mehta RL: Automated electronic monitoring of circuit pressures during continuous renal replacement therapy: a technical report. Blood Purif 2001;19:227-232.

28 Kim IB, Fealy N, Baldwin I, Bellomo R: Premature circuit clotting due to likely mechanical failure during continuous renal replacement therapy. Blood Purif 2010;30:79-83.

29 Dunn WJ, Sriram S: Filter lifespan in critically ill adults receiving continuous renal replacement therapy: the effect of patient and treatment-related variables. Crit Care Resusc 2014;16:225-231.

30 Rewa O, Mottes T, Bagshaw SM: Quality measures for acute kidney injury and continuous renal replacement therapy. Curr Opin Crit Care 2015;21:490-499.

31 Ronco C: Fluid balance in CRRT: a call to attention! Int J Artif Organs 2005;28:763-764. 
32 Bagshaw SM, Baldwin I, Fealy N, Bellomo R: Fluid balance error in continuous renal replacement therapy: a technical note. Int J Artif Organs 2007;30:434-440.

33 Bouchard J, Soroko SB Chertow GM, Himmelfarb J, Ikizler TA, Paganini EP, et al: Fluid accumulation, survival and recovery of kidney function in critically ill patients with acute kidney injury. Kidney Int 2009;76:422-427.

34 Rewa O, Villeneuve PM, Eurich DT, Stelfox HT, Gibney RT, Hartling L, et al: Quality indicators in continuous renal replacement therapy (CRRT) care in critically ill patients: protocol for a systematic review. Syst Rev 2015;4:102.

35 Graham P, Lischer E: Nursing issues in renal replacement therapy: organization, manpower assessment, competency evaluation and quality improvement processes. Semin Dial 2011;24:183-187.

36 Sutherland SM, Goldstein SL, Alexander SR: The prospective pediatric continuous renal replacement therapy (ppCRRT) registry: a critical appraisal. Pediatr Nephrol 2014;29: 2069-2076.

37 Honore PM, Jamez J, Wauthier M, Lee PA, Dugernier T, Pirenne B, et al: Prospective evaluation of short-term, high-volume isovolemic hemofiltration on the hemodynamic course and outcome in patients with intractable circulatory failure resulting from septic shock. Crit Care Med 2000;28:3581-3587.

38 Ratanarat R, Brendolan A, Piccinni P, Dan M, Salvatori G, Ricci Z, et al: Pulse high-volume haemofiltration for treatment of severe sepsis: effects on hemodynamics and survival. Crit Care 2005;9:R294-R302.
39 Joannes-Boyau O, Honoré PM, Perez P, Bagshaw SM, Grand H, Canivet JL, et al: Highvolume versus standard-volume haemofiltration for septic shock patients with acute kidney injury (IVOIRE study): a multicentre randomized controlled trial. Intensive Care Med 2013;39:1535-1546.

40 Zhang P, Yang Y, Lv R, Zhang Y, Xie W, Chen $\mathrm{J}$ : Effect of the intensity of continuous renal replacement therapy in patients with sepsis and acute kidney injury: a single-center randomized clinical trial. Nephrol Dial Transplant 2012;27:967-973.

41 Clark E, Molnar AO, Joannes-Boyau O, Honoré PM, Sikora L, Bagshaw SM: High-volume hemofiltration for septic acute kidney injury: a systematic review and meta-analysis. Crit Care 2014;18:R7.

42 Cruz DN, Perazella MA, Bellomo R, de Cal M, Polanco N, Corradi V, et al: Effectiveness of polymyxin B-immobilized fiber column in sepsis: a systematic review. Crit Care 2007; 11:R47.

43 Cruz DN, Antonelli M, Fumagalli R, Foltran F, Brienza N, et al: Early use of polymyxin B hemoperfusion in abdominal septic shock: the EUPHAS randomized controlled trial. JAMA 2009;301:2445-2452.

44 Payen DM, Guilhot J, Launey Y, Lukaszewicz AC, Kaaki M, Veber B, et al: Early use of polymyxin $B$ hemoperfusion in patients with septic shock due to peritonitis: a multicenter randomized control trial. Intensive Care Med 2015;41:975-984.

45 Morgera S, Haase M, Kuss T, Vargas-Hein O, Zuckermann-Becker H, Melzer C, et al: Pilot study on the effects of high cutoff hemofiltration on the need for norepinephrine in septic patients with acute renal failure. Crit Care Med 2006;34:2099-2104.
46 Honoré PM, Jacobs R, Boer W, Joannes-Boyau O, De Regt J, De Waele E, et al: New insights regarding rationale, therapeutic target and dose of hemofiltration and hybrid therapies in septic acute kidney injury. Blood Purif 2012;33:44-51.

47 Taniguchi T: Cytokine adsorbing columns 2010;166:134-141.

48 Peng ZY, Carter MJ, Kellum JA: Effects of hemoadsorption on cytokine removal and short-term survival in septic rats. Crit Care Med 2008;36:1573-1577.

49 Quintel M: CytoSorb ${ }^{\mathrm{TM}}$ Whole Blood Cytokine Adsorption - Results of a Controlled Randomized Trial. Brussels, 32nd International Symposiumon Intensive Care and Emergency Medicine, 2012.

50 Combes A, Brodie D, Bartlett R, Brochard L, Brower R, Conrad S, et al: Position paper for the organization of extracorporeal membrane oxygenation programs for acute respiratory failure in adult patients. Am J Respir Crit Care Med 2014;190:488-496.

51 Jacobs R, Honore PM, Spapen HD: Intertwining extracorporeal membrane oxygenation and continuous renal replacement therapy: sense or nonsense? Crit Care 2015;19: 145 .

52 Terragni P, Maiolo G, Ranieri VM: Role and potentials of low-flow $\mathrm{CO}(2)$ removal system in mechanical ventilation. Curr Opin Crit Care 2012;18:93-98.

53 Yamashita A, Masaki H, Kobayashi E, Sukegawa $\mathrm{T}$ : Evaluation of solute penetration across the polysulfone membrane with vitamin E coating. Hemodial Int 2015;19(suppl 3):S20-S25. 